\title{
Hydrolyzed Collagen-Based Hydrogel System Design, Characterization and Application in Drug Delivery
}

\author{
Palapparambil Sunny Gils ${ }^{\S *}$, Nalin Kumar Sahu ${ }^{\S b}$, Debajyoti Ray ${ }^{\S c}$, Prafulla Kumar Sahoo ${ }^{a}$
}

${ }^{a}$ Polymer Research Unit, Department of Chemistry, Utkal University, Vani Vihar, Bhubaneswar, India

${ }^{b}$ Polymer Research Unit, Department of Chemistry, M.P. Mahavidyalaya College, Erakana, India

${ }^{c}$ P.G. Department of Pharmaceutics, Sri Jayadev College of Pharmaceutical Sciences, Bhubaneswar, India

$\S$ : These authors contributed equally to this work.

*Corresponding Author:

Palapparambil Sunny Gils

Polymer Research Unit

Department of Chemistry

Utkal University

Vani Vihar, Bhubaneswar 751004

India

Email: gils.ps@gmail.com

Received: 5 November 2011; | Revised: 4 February 2012; | Accepted: 21 February 2012

\begin{abstract}
This work was intended to develop a new hydrogel of collagen-g-Poly (acrylamide-co-itaconic acid) through chemical cross-linking by graft copolymerization of acrylamide (AM) and itaconic acid (IA) on to collagen (CGN) via redox initiator system of ammonium persulfate (APS) and $N, \quad N, N^{\prime}, N^{\prime}-$ tetramethylethylenediamine (TMED), in presence of $N, N^{\prime}$-methylene bis acrylamide (MBA) as crosslinking agent. Characterization of the hydrogel was done by FT-IR, TGA, SEM, LCMS/MS and HPLC. Valsartan (VAL) was successfully loaded into the prepared hydrogel. CGN-g-P(AM-co-IA) $\left(\mathrm{H}_{10}\right)$ formulation showed highest swelling capacity as well as VAL release in the biological media and release was controlled up to 24h. The release data of various formulations were fitted to Zero order, First order and Higuchi's kinetic models. It was observed that the release of drug from all the formulations followed Higuchi's kinetic model as its value of coefficient of determination is greater than that of others.
\end{abstract}

Keywords: Hydrogel, Collagen, Acrylamide, Itaconic acid, Residual monomers, Valsartan delivery.

\section{Introduction}

Hydrogels are lightly crosslinked networks of hydrophilic polymer chains, which can retain large amount of water within its structure but do not dissolve into it. Polymers from natural, synthetic or semi-synthetic sources can be used for preparing hydrogels. It involves crosslinking of either linear polymers or simultaneous polymerization and crosslinking of monomers with polyfunctional monomers 1,2 . The most 
effective hydrogels are obtained from polymers having dissociative ionic functional groups. The presence of ionic groups in the hydrogel structure changes its charge state against changes in $\mathrm{pH}$ and ionic strength of the medium. The absorbent properties of polymeric hydrogels have been commercially exploited in hygiene products, agricultural and site specific controlled drug delivery applications. At present, stimuli sensitive drug delivery systems (DDSs) have been an attractive theme for controlled release ${ }^{3,4}$. The release behaviors of drugs can be easily controlled by surrounding properties, such as $\mathrm{pH}$, temperature, ionic strength and electric field. As compared with conventional administration, drugs can prolong their duration time by hydrogel DDSs.

Collagen (CGN) is the most abundant protein in animal kingdom as it represents close to $30 \%$ of mammalian protein matter. Because of its spatial structure and high molecular weight, native collagen is naturally insoluble in water. In order to be separated from the other constituents of animal tissues, it is made soluble through an extraction process, which includes first a partial and controlled hydrolysis of the protein chain and then a warm water extraction. This yields hydrolyzed collagen. In nutraceuticals, hydrolyzed collagen could be incorporated in various "dosage forms". Numerous hydrogels composed of synthetic acrylic derivatives like hydroxyethyl methacrylate, acrylic acid, methacrylic acid, Nisopropylacrylamide, and natural polysaccharides like chitosan exhibit $\mathrm{pH}$-sensitive behavior and subsequently used for colonic drug delivery ${ }^{5-9}$. There are various copolymers of itaconic acid which also shows the $\mathrm{pH}$-sensitive swelling and drug release behavior. Therefore, the diseases of the colon are treated through direct delivery of the drugs to the site of action. Itaconic acid is one of the monomers, which is readily available at low cost. It is obtained from renewable resources by fermentation with Aspergillus terrus using carbohydrate materials as molasses and hydrolyzed starch ${ }^{10}$. Itaconic acid easily copolymerizes and provides polymer chains with carboxylic side groups, which are highly hydrophilic and able to form hydrogen bonds with corresponding groups. Unlike monocarboxylic methacrylic and acrylic acid, itaconic acid has two
$-\mathrm{COOH}$ groups with different $\mathrm{pKa}\left(\mathrm{pKa}_{1}=3.85\right.$ and 5.45) values, so that very small amounts of IA render good $\mathrm{pH}$ sensitivity and increased degree of hydrogel swelling. In addition, incorporation of co-monomers which can contribute to H-bonding can increase the mechanical strength of the hydrogel. Furthermore, IA is very hydrophilic and is expected to show high biocompatibility because of its natural source.

The purpose of this study was to synthesize intelligent CGN-based hydrogels to be used as $\mathrm{pH}$ sensitive carriers for the controlled delivery of valsartan (VAL), a long-acting angiotensin receptor blocker (ARB). VAL was used as model drug because it is absorbed at different sites in the human gastrointestinal tract ${ }^{11}$.

\section{Experimental}

\subsection{Materials Materials}

Hydrolyzed collagen (CGN) (Himedia Laboratories Pvt. Ltd., Mumbai) is of industrial grade which is available in market and has nearly $25 \%$ insoluble phosphate salt. Acrylamide (AM), Ammonium persulfate (APS), $N, N, N$ 'N'-tetramethylethylenediamine (TMED), $N, N$ 'methylene-bisacrylamide (MBA) (Merk) were used without further purification. Itaconic acid was purchased from fluka and used as received. Orthophosphoric acid from E-MERK, Germany. Acrylamide was supplied by Sigma Chemical Company (St. Louis, MO, USA). Acrylamide-d3 was purchased from Polymer Source Inc. (Dorval, Quebec, Canada). Individual stock standard solutions of $100 \mathrm{mg} / \mathrm{l}$ in water were prepared and stored at $4^{0} \mathrm{C}$ for 6 months. Analytically pure valsartan was purchased from Fluka AG, Chemische Febrik, Switzerland. All other chemicals used in this study were of analytical grade quality, with deionized water (milli Q, Millipore (India) Pvt Ltd, Bangalore) being used for preparing the solutions.

\subsection{Synthesis}

Synthesis of Collagen-g-Poly (acrylamide-coitaconic acid), CGN-g-Poly (AM-co-IA)

A pre-weighed amount of hydrolyzed CGN $(1.0 \mathrm{~g})$ was added to $50 \mathrm{ml}$ deionized water and filtered to remove its insoluble phosphate salt. 
Then the solution was added to a three-necked 500 $\mathrm{ml}$ reactor equipped with a mechanical stirrer (RZR 2021), a three-blade propeller (Heidolph, Schwabach, Germany) and stirred at $250 \mathrm{rpm}$ for $10 \mathrm{~min}$. The reactor was placed in a thermostated water bath to control the reaction temperature at $80{ }^{\circ} \mathrm{C}$. After dissolving and homogenizing the mixture, the monomers AM, IA and the crosslinker, $\mathrm{MBA}^{12}$ were simultaneously added and the reaction mixture was stirred for $15 \mathrm{~min}$. Then the initiator APS (oxidant) and TMED (reductant) were added (Table I). The solution was stirred at 400-500 rpm while maintaining the temperature and inert atmosphere. The temperature was maintained at $80^{\circ} \mathrm{C}$ and the reaction mixture was stirred continuously for $24 \mathrm{~h}$. The low molecular weight substances remaining in the samples after polymerization were extracted with boiling ethanol for $24 \mathrm{~h}$. The product was collected by centrifugation and dried in the oven under vacuum at $60{ }^{\circ} \mathrm{C}$ for $24 \mathrm{~h}$. The dried graft polymer was added to $300 \mathrm{ml}$ deionized water. It was allowed to swell during agitation in a water bath at the constant temperature of $60^{\circ} \mathrm{C}$ for $24 \mathrm{~h}$. Then it was extracted with ethanol in a soxhlet for $6 \mathrm{~h}$ followed by water at $100{ }^{\circ} \mathrm{C}$ for $72 \mathrm{~h}$. The Hydrogel was filtered and dried under vacuum at $60^{\circ} \mathrm{C}$. After grinding, the resulting powder was stored away from moisture, heat and light. Homopolymer, PAM (poly acrylamide) and copolymer and copolymer Poly (AM-co-IA) were also synthesized using the same method and by FT-IR studies (Fig. 1).

Table I Composition of the feed mixture

\begin{tabular}{|c|c|c|c|c|c|}
\hline $\begin{array}{c}\text { Polymer } \\
\text { code }\end{array}$ & $\begin{array}{c}\text { IA } \\
(\mathbf{m g})\end{array}$ & $\begin{array}{c}\text { MBA 1\% (w/v) } \\
\text { ml }\end{array}$ & $\begin{array}{c}\text { TMED 1\% (w/v) } \\
\text { ml }\end{array}$ & $\begin{array}{c}\text { APS 1\% (w/v) } \\
\text { ml }\end{array}$ & \% Yield \\
\hline H1 & 0 & 0.4 & 0.3 & 0.2 & 51 \\
\hline H2 & 20 & 0.4 & 0.3 & 0.2 & 54 \\
\hline H3 & 40 & 0.4 & 0.3 & 0.2 & 61 \\
\hline H4 & 60 & 0.4 & 0.3 & 0.2 & 78 \\
\hline H5 & 80 & 0.4 & 0.3 & 0.2 & 58 \\
\hline H6 & 60 & 0.2 & 0.3 & 0.2 & 62 \\
\hline H7 & 60 & 0.6 & 0.3 & 0.2 & 59 \\
\hline H8 & 60 & 0.8 & 0.3 & 0.2 & 54 \\
\hline H9 & 60 & 0.4 & 0.1 & 0.2 & 63 \\
\hline H10 & 60 & 0.4 & 0.2 & 0.2 & 92 \\
\hline H11 & 60 & 0.4 & 0.4 & 0.2 & 79 \\
\hline H12 & 60 & 0.4 & 0.2 & 0.1 & 70 \\
\hline H13 & 60 & 0.4 & 0.2 & 0.3 & 72 \\
\hline H14 & 60 & 0.4 & 0.2 & 0.4 & 73 \\
\hline
\end{tabular}

\subsection{Characterization}

FTIR spectra of individual and crosslinked polymers were recorded in the range 400-4000 $\mathrm{cm}^{-1}$ on a Perkin Elmer Paragon 500 FTIR spectrophotometer using $\mathrm{KBr}$ pellets. The thermogravimetric analysis data were recorded with a Shimadzu DTG-50 thermal analyzer. The samples were heated from room temperature to $600^{\circ} \mathrm{C}$ at a heating rate of $10^{\circ} \mathrm{C}$ per min. The SEM of gold-coated samples were obtained using JSM - 6390LV scanning electron microscope (Jeol Ltd,
Japan) at a magnification of $\mathrm{x} 5$ to 300,000 (Resolution-HV $3.0 \mathrm{~nm}$ ). Residual AM was detected and quantified by Liquid Chromatography Mass Spectrometry (LCMS/MS). Analysis was performed on a Perkin-Elmer 200 Micro pump series system (perkin-Elmer, Uberlingen, Germany) coupled to an Applied Biosystem API 2000 triple quadrupole mass spectrometer equipped with a Turboionspary ionization source (Applied Biosystem, Foster City, CA). MS detection was performed in the positive 
ion mode using multiple reactions monitoring (MRM). Data was acquired and processed by Analyst software (version 1.4.1). Residual IA was detected and quantified by HPLC (Prominence, Shimadzu Corporation, Japan). The chromatographic system consisted of a computercontrolled pump (model LC 20AT), autosampler (model SIL-10AF) equipped with a $200 \mu 1$ sample loop, photodiode array (PDA) detector (model SPD-M20A). Shimadzu LC Solution software was used for the system and data management. The separation was performed in isocratic mode at a flow rate of $1.0 \mathrm{ml} / \mathrm{min}$ and a temperature of 40 ${ }^{\circ} \mathrm{C}$ on an analytical column Gemini $5 \mu \mathrm{C} 18,150 \mathrm{x}$ $4.6 \mathrm{~mm}$ (Phenomenex, USA). An RP C18 Security guard (4 x 3mm, Phenomenex) was employed to protect the analytical column. The mobile phase was aqueous $0.05 \%$ orthophosphoric acid and the injection volume was 50 4 . The observed backpressure values were in the range from 1400 to $1450 \mathrm{psi}$. Data was acquired and processed by LC solution software (Shimadzu, Japan).

\subsection{Swelling behavior of CGN-g-P (AM-co-IA)}

Swelling behavior of CGN-g-Poly (AM-coIA) was performed by tea bag ${ }^{13}$ method. About $0.100 \mathrm{~g}$ of sample was added to a small bag made of nylon (50 mm x $90 \mathrm{~mm} ; 200 \mathrm{mesh})$. Then the bag was completely immersed in the swelling medium $(200 \mathrm{ml})$ at room temperature for $24 \mathrm{~h}$ to reach the swelling equilibrium. The $\mathrm{pH}$-dependent equilibrium swelling of the hydrogel was studied both in the simulated gastric fluid (SGF, 3.2 $\mathrm{mg} / \mathrm{ml}$ pepsin in $0.05 \mathrm{M}$ hydrochloric acid, $\mathrm{pH}$ 1.2) and simulated intestinal fluid (SIF, $10 \mathrm{mg} / \mathrm{ml}$ pancreatin in Sorensen's phosphate buffer, $\mathrm{pH}$ 7.4). Adhered liquid droplets on the surface of the particles were removed by blotting with tissue papers. The swollen hydrogels were weighed and dried in an oven at $60{ }^{\circ} \mathrm{C}$ for $6 \mathrm{~h}$ until there was no change in the dry mass of the samples.

The \% equilibrium swelling (ES) was defined as follows:

$$
\mathrm{ES}(\%)=\left(\mathrm{W}_{\mathrm{S}^{-}} \mathrm{W}_{\mathrm{d}}\right) \times 100
$$

Where $\mathrm{W}_{\mathrm{S}}$ and $\mathrm{W}_{\mathrm{d}}$ are the weights of the swollen sample and the weight of dried gel, respectively.

\subsection{Drug loading}

CGN-g-Poly(AM-co-IA) was loaded with VAL (as model drug) by soaking in an aqueous solution containing $10 \%(\mathrm{w} / \mathrm{v})$ of VAL. Soaking was done for nearly 2 days in order to achieve complete equilibrium. The formulations were filtered and the surface-adhered drug solution was removed by washing and blotting with soft filter paper and dried in air before storing in a desiccator.

\subsection{Drug entrapment efficiency (\%)}

VAL loaded CGN-g-Poly (AM-co-IA) (50 $\mathrm{mg}$ ) from each batch were dispersed separately in water and kept for $24 \mathrm{~h}$, filtered through $0.22 \mu \mathrm{m}$ microfilter and absorbance was measured using UV/VIS spectrophotometer (Varian, Cary 50 Bio,USA) at $250 \mathrm{~nm}$. The obtained absorbance was plotted on the standard curve to get the exact concentration of the entrapped drug. VAL content were determined and expressed in terms of weight of VAL per weight of hydrogel, thus determining the actual entrapment ratio (AER).

$\%$ of Entrapment efficiency $=$ AER $/$ TER $\times 100$

where AER = Measured drug wt $/$ formulation wt and

TER = Drug wt. /drug wt. and polymer wt.

\subsection{In vitro drug release study in $\mathrm{pH}$ progressive media}

The in vitro drug release was carried out by filling the calculated amount of VAL loaded CGN-g-Poly (AM-co-IA) in capsule shell (size 2), analyzed using USP-I basket dissolution apparatus and proper simulation of gastro intestinal (GIT) condition was maintained by altering the $\mathrm{pH}$ of dissolution medium at different time intervals following two step-dissolution conditions. To simulate the physiological conditions of GIT, first $2 \mathrm{~h}$ of dissolution were carried out in $900 \mathrm{ml}$ of simulated gastric fluid (SGF, $3.2 \mathrm{mg} / \mathrm{ml}$ pepsin in $0.05 \mathrm{M}$ hydrochloric acid, $\mathrm{pH}$ 1.2) and the rest of the time in $900 \mathrm{ml}$ of simulated intestinal fluid (SIF, $10 \mathrm{mg} / \mathrm{ml}$ pancreatin in Sorensen's phosphate buffer, $\mathrm{pH}$ 7.4). The media was stirred at $100 \mathrm{rpm}$ at $37 \pm 0.5$ ${ }^{\circ} \mathrm{C}$. At predetermined time intervals, specified amount of dissolution medium was removed; 
filtered through $0.22 \mu \mathrm{m}$ microfilter and analyzed in UV spectrophotometer at $250 \mathrm{~nm}$. After each sampling, an equal volume of fresh dissolution media was added to the dissolution medium. All the dissolution studies were repeated six times.

\subsection{Kinetics of drug release}

Different mathematical models may be applied for describing the kinetics of the drug release process from the CGN-g-Poly (AM-co-IA) hydrogel matrix; the most suited being the one which best fits the experimental results. The kinetics of VAL release from hydrogel was determined by finding the best fit of the dissolution data (drug release $v s$. time) to distinct models: Zero order [eq.1], first-order [eq.2] and Higuchi [eq. 3].

$\mathrm{Q}_{\mathrm{t}}=\mathrm{k}_{0} \mathrm{t}[1]$

$\mathrm{Q}_{\mathrm{t}}=\mathrm{Q}_{\infty}\left(1-\mathrm{e}^{-\mathrm{k}}{ }_{1}^{\mathrm{t}}\right)[2]$

$\mathrm{Q}_{\mathrm{t}}=\mathrm{k}_{\mathrm{H}} \mathrm{t}^{1 / 2}[3]$

where $\mathrm{Q}_{\infty}$ being the total amount of drug in the matrix, $\mathrm{k}_{0}$ the zero order kinetic constant, $\mathrm{k}_{1}$ the first order kinetic constant and $\mathrm{k}_{\mathrm{H}}$ representing the Higuchi rate constant.

\subsection{Stability studies}

To assess the physical stability, selected VAL delivery systems (H4, H10 and H11) were stored in the stability chamber at $40{ }^{\circ} \mathrm{C} / 75 \%$ relative humidity condition as per I.C.H. guidelines ${ }^{14}$. Samples of definite amount from each batch were withdrawn after three months to see the effect of VAL release from the gel on storage.

\section{Results and Discussions}

\subsection{FT-IR spectra}

Fig. 1 shows the FTIR spectra of PAM, Poly (AM-co-IA), CGN-g-Poly (AM-co-IA), VAL, VAL incorporated into graft copolymer. For CGN-g-Poly (AM-co-IA) (c), the peaks found at 3401, 1649, and $1600 \mathrm{~cm}^{-1}$ indicate the $\mathrm{N}-\mathrm{H}$ stretching, the $\mathrm{C}=\mathrm{O}$ stretching and $\mathrm{N}-\mathrm{H}$ bending of the amide bands, respectively, which are characteristics of the $-\mathrm{CONH}_{2}$ group containing in the acrylamide. In addition, the peak at 1390 $\mathrm{cm}^{-1}$ is for the $-\mathrm{C}-\mathrm{N}$ stretching and $599 \mathrm{~cm}^{-1}$ for the weak band $\mathrm{N}-\mathrm{H}$ out of plane bending. These are the typical absorption bands of the amide. The broad band at $3200-3600 \mathrm{~cm}^{-1}$ is due to the overlapping of $\mathrm{N}-\mathrm{H}$ stretching band of amide with $-\mathrm{OH}$ stretching band of the collagen portion of the graft copolymer. Moreover, band at $1711 \mathrm{~cm}^{-1}$ was assigned to carboxylic carbonyl group of itaconic acid. This is in good agreement with the results reported in the literature ${ }^{15}$. VAL alone showed two carbonyl absorption bands at 1729 and 1609 $\mathrm{cm}^{-1}$, assigned to the carboxyl carbonyl and amide carbonyl stretching, respectively (d). These bands are of indicative value to elucidate drug-polymer interactions. The two absorption bands of the pure drug appeared unchanged in the graft copolymer (e).

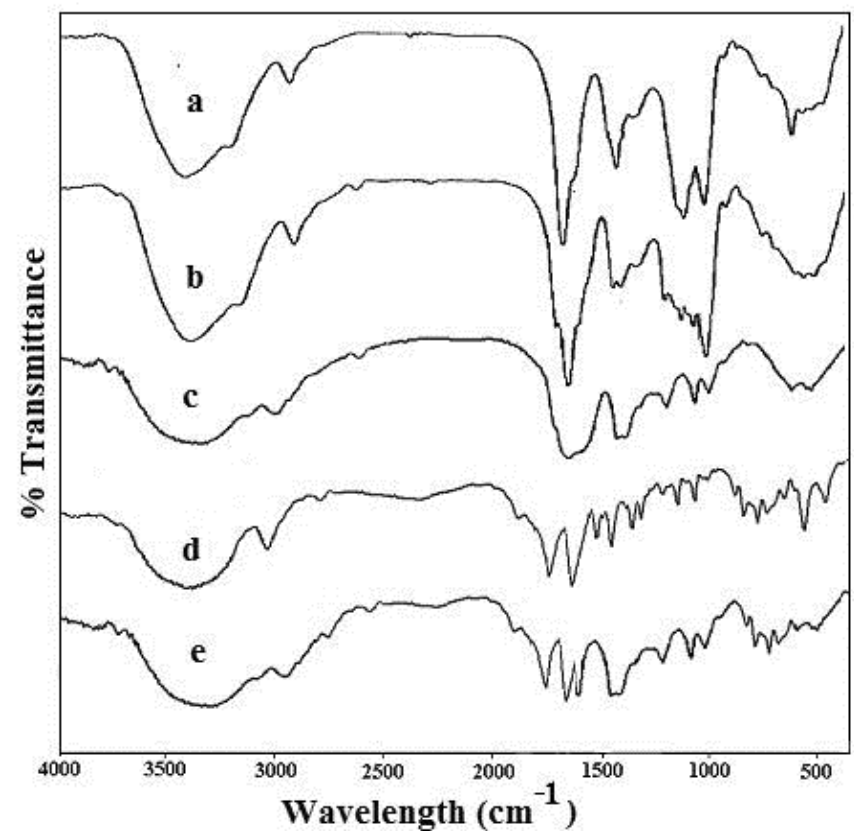

Figure 1. FTIR spectra of PAM (a), Poly (AM-co-IA) (b), CGN-g-Poly (AM-co-IA) (c), VAL (d) VAL incorporated into graft copolymer (e).

\subsection{Thermal analysis}

The results of thermogravimetric analysis (TGA) technique employed to characterize the thermal properties of the obtained graft copolymers as shown in Fig. 2. CGN (a) shows a two-step characteristic thermogram, wherein the major weight loss $(73 \%)$ takes place in the second step within the temperature range of $176-334{ }^{\circ} \mathrm{C}$, the temperature for a maximum decomposition was $318^{\circ} \mathrm{C}$. The thermogram of Poly (AM-co-IA) (b) showed three decomposition stages. The first decomposition stage in the range $38-110{ }^{\circ} \mathrm{C}$ was 
attributed to the loss of bound water. The second one in the interval of $181.1-292{ }^{\circ} \mathrm{C}$ had been described to the decarboxylation of IA coupled with the chain scission. The Weight loss in the third or main stage of decomposition $\left(295-387^{\circ} \mathrm{C}\right)$ can be assigned to the degradation of acrylamide portion. In case of CGN grafted with Poly (AMco-IA) (c), four stages of decomposition were observed. It is suggested that in an initial stage of the thermal diagram, when the temperature in a range from ambient temperature to about $150{ }^{\circ} \mathrm{C}$, the weight loss is a result of the dehydration process of the water contained in such a hydrophilic polymer. At the second stage from 151 to $269^{\circ} \mathrm{C}$, there is a decomposition peak in the side groups and branches of the graft copolymer (carboxyl group in itaconic acid proportion). At the third stage from 270 to $337{ }^{\circ} \mathrm{C}$, there is a degradation of $\mathrm{CGN}$ in the graft copolymer. However, at the fourth stage about $338{ }^{\circ} \mathrm{C}$, the weight loss was found as a result of the degradation of the polymer chain and matrices (degradation of polysaccharide and acrylamide portion). From the TGA curves, it can be concluded that the thermal stability of the polysaccharide decreases with the grafting of Poly (AM-co-IA) chains onto the polysaccharide backbone. This may be attributed to the low thermal stability of CGN and itaconic acid. Similar phenomenon has also been reported by $\mathrm{N}$. Isıklan ${ }^{14}$. He has indicated that thermal stability of the polymer reduced with the grafting of IA onto sodium alginate.

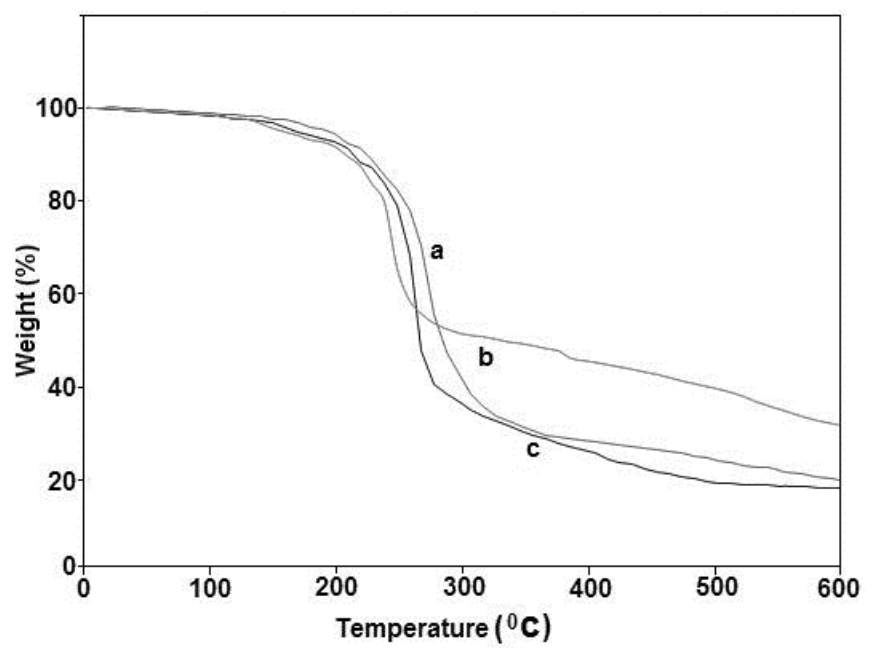

Figure 2. TGA of (a) CGN (b) Poly (AM-co-IA) (c) CGN-g-Poly (AM-co-IA) [H10].

\subsection{Scanning Electron Microscopy}

Scanning electron microscopy (Fig. 3) allows much high magnification of the hydrogel structure in which we can see the surface. This picture verifies that the graft copolymers prepared in this work have a porous structure. The surface of the hydrogel is rougher and the approximate diameter of the pores was found to be in between 50 to 150 $\mu \mathrm{m}$. The uneven surface may be due to a quite high viscosity of the gel and solvent evaporation process. It is supposed that these pores are the regions of water permeation and interaction sites of external stimuli with the incorporated drug or hydrophilic.
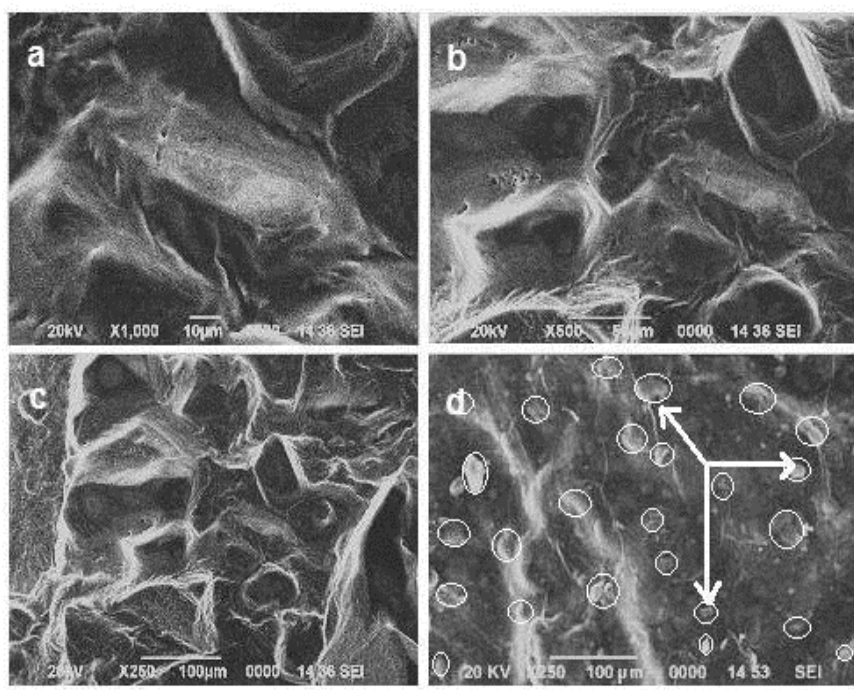

Figure 3. SEM image of the CGN-g-Poly (AM-co-IA) [H10] at different magnifications $(\mathrm{a}, \mathrm{b}$ and $\mathrm{c})$, VAL loaded H10 (d).

groups of the graft copolymers. Therefore, the porous structure is the predominant reason for the higher swelling rate. The results show that the drug is uniformly distributed in the hydrogel (Fig. $3 \mathrm{~d})$.

\subsection{Residual AM determination}

Dried xerogel $(0.100$ g) was accurately weighed and added to $4.5 \mathrm{ml}$ water in an amber vial (Supleco, USA). $500 \mu \mathrm{l}$ of the internal standard (100 ng/ml) solution, d3-acrylamide, was added. After vortex (Cyclo mixer, CM 101, Remi Instruments, India) for $10 \mathrm{~min}$ the sample hydrogels were placed in an orbital shaker (Labline instruments, India) at $37^{\circ} \mathrm{C}$ with constant agitation $(200 \mathrm{rpm})$ for $24 \mathrm{~h}$. Then it was 
centrifuged (model 510R, Eppendorf, Germany) for $10 \mathrm{~min}$ at $4000 \mathrm{rpm}$ at $5^{\circ} \mathrm{C}$. The samples are respectively shaked during $1 \mathrm{~min}$ on a Vortex and $10 \mathrm{~min}$ by orbital rotation. The extract is centrifuged at $5{ }^{\circ} \mathrm{C}$ with a speed of $4000 \mathrm{rpm}$; the supernatant was collected filtered on a nylon membrane. The supernatant was passed through Oasis ${ }^{\circledR} \quad$ HLB SPE cartridges previously conditioned with $5 \mathrm{ml}$ of methanol and $5 \mathrm{ml}$ of water. Elution occurs with $5 \mathrm{ml}$ of water. A second SPE purification involves the Bond ElutAccucat ${ }^{\circledR}$ cartridges conditioned with $5 \mathrm{ml}$ of methanol and $5 \mathrm{ml}$ of water before loading with the totality of the extract. Eluent was directly collected and filtered. $20 \mu \mathrm{l}$ was injected into LCMS/MS.


Figure 4. LCMS/MS Chromatograms. (a) Blank (Methanol), (b) AM standard $0.250 \mu \mathrm{g} / \mathrm{ml}$, (c) AM in sample (H10) (before extraction),(d) AM in sample (H10) (after soxhlet extraction).
For the detection by LC-MS/MS, identification occurs with the relative retention time (RRT) and diagnostic ions consisting mainly of the precursor ion at $\mathrm{m} / \mathrm{z}, 72.04$ and one daughter ion (quantifier) resulting from a loss of $\mathrm{HCN}$ at $m / z \quad 55.10^{16}$. For confirmatory purpose, a comparison with quality control samples were made using acceptable deviations of $\pm 2.5 \%$ for relative retention time and $\pm 20 \%$ for the ionic relative abundance as described in the European

Commission Decision 2002/657/EC. It can be seen from the Fig. 4 that the amount of the residual AM was decreased markedly after soxhlet extraction (the amount was decreased from 28.41 $\mu \mathrm{g} / \mathrm{g}$ to $0.503 \mu \mathrm{g} / \mathrm{g}$ ). The decrease in residual AM content was due to its diffusion from gel network to the water, as it was allowed to swell in the excess of water, but a little amount was still detected as few monomers were trapped in the polymer chain.

\subsection{Residual IA determination}

Powdered hydrogel sample $(0.300 \mathrm{~g})$ was accurately weighed and added to $10 \mathrm{ml}$ methanolic orthophosphoric acid $(10: 90, \mathrm{pH}=2.3)$ in a polypropylene tube. After vortex (Cyclo mixer, CM 101, Remi Instruments, India) for 10 minutes the sample hydrogels were placed in an ultrasonicator bath ( Toshcon, SW-7, India) for half an hour followed by placing in an orbital shaker (Labline instruments, India) at $37{ }^{\circ} \mathrm{C}$ with constant agitation (200 rpm) for $12 \mathrm{~h}$. Then it was centrifuged (Eppendorf, 510R, Germany) for 10 minutes at $3500 \mathrm{rpm}$ at $4{ }^{\circ} \mathrm{C}$. The supernatant was taken by means of a syringe, then filtered through a $0.45 \mu \mathrm{m}$ syringe filter (Millipore millex-HV, Hydrophillic PVDF) and finally put in a sample vial (Waters,USA). The PDA absorbance over the 205-500 nm range was recorded and the wavelength used for quantification was $210 \mathrm{~nm}$.

Standard solutions of IA $(0.1,0.2,0.4,0.6$, 0.8 and $1.0 \mathrm{mg} / \mathrm{ml}$ ), each in five replicates were injected into the system. Linearity of the analytical procedure was evaluated by plotting detector response (peak area) against analyte concentration. The regression equation was $\mathrm{Y}=\mathrm{a} \mathrm{X}+\mathrm{b}$, where $\mathrm{Y}$ denotes peak area and $\mathrm{X}$ is the concentration of IA $(\mu \mathrm{g} / \mathrm{ml})$. The $\mathrm{r}^{2}$ values for IA was found to be $>0.9990$, confirming the linear relationship 
between the concentration of the IA and area under the curve. Methanolic orthophosphoric acid (10:90) was used as extraction solvent. The coiled and packed chains of hydrogel matrix unfold and make rooms or voids for solvent molecules as it was allowed to swell and the total residual monomer in form of either acid or its salt diffuses from gel network to the extracting solution. Representative HPLC chromatograms of itaconic acid in different hydrogel matrix are shown in Fig. 5. In this case also residual IA graft co-polymer decreased considerably after soxhlet extraction i.e. from $24.01 \mu \mathrm{g} / \mathrm{g}$ to $7.33 \mu \mathrm{g} / \mathrm{g}$.
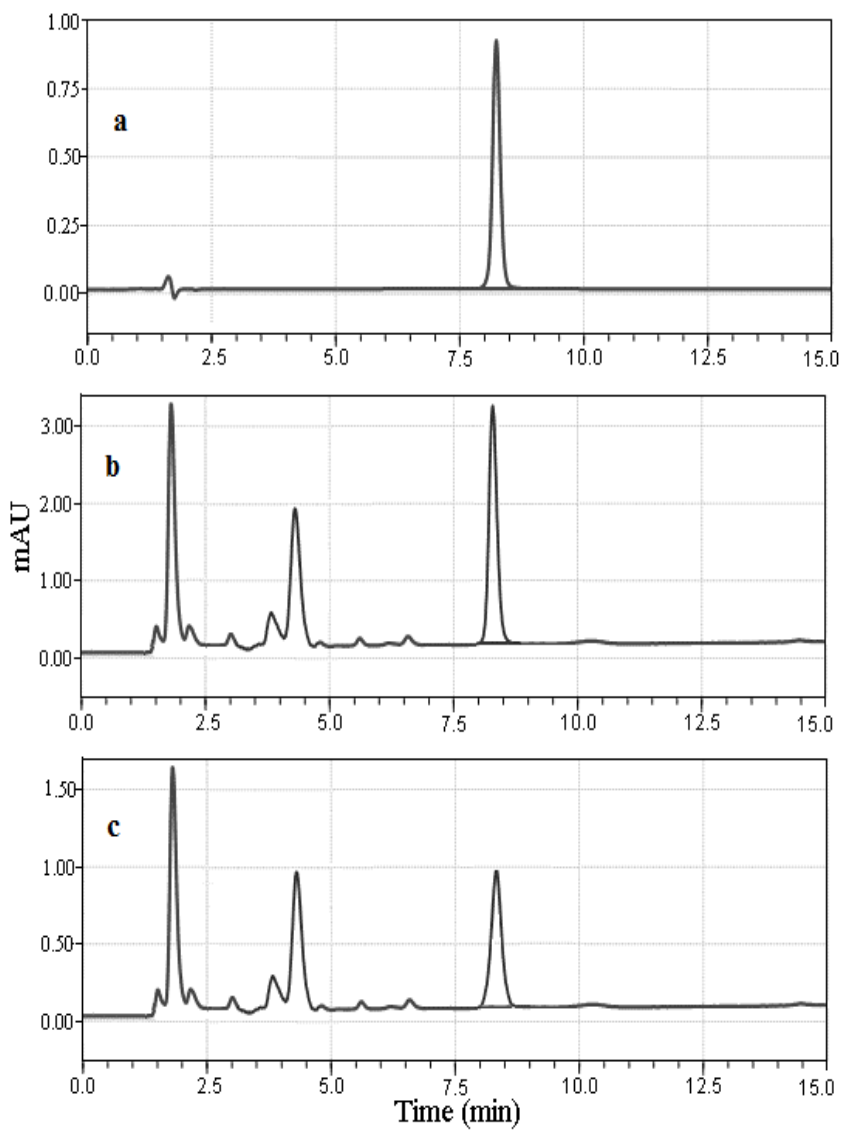

Figure 5. Representative HPLC chromatograms of standard itaconic acid $(0.2 \mu \mathrm{g} / \mathrm{ml})$ (a), residual itaconic acid detected in CGN-g-Poly (AM-co-IA) (before soxhlet extraction) (b), after soxhlet extraction (c).

\subsection{Swelling behavior}

Swelling studies were performed in simulated gastric fluid (SGF) and simulated intestinal fluid (SIF) as shown in Fig. 6. From the study, swelling of hydrogels in SIF media was found to be much higher than that in SGF. Under acidic $\mathrm{pH}$ values
(SGF), most of the carboxylate anions were protonated. So the main anion-anion repulsive forces were eliminated and consequently swelling values were decreased. At higher $\mathrm{pH}$ values (SIF), some of the carboxylate groups were ionized and the electrostatic repulsion between $\mathrm{COO}^{-}$groups caused an enhancement of the swelling capacity. CGN-g-P (AM-co-IA) (H10) showed slightly highest swelling capacity in both the media compared to other hydrogels.

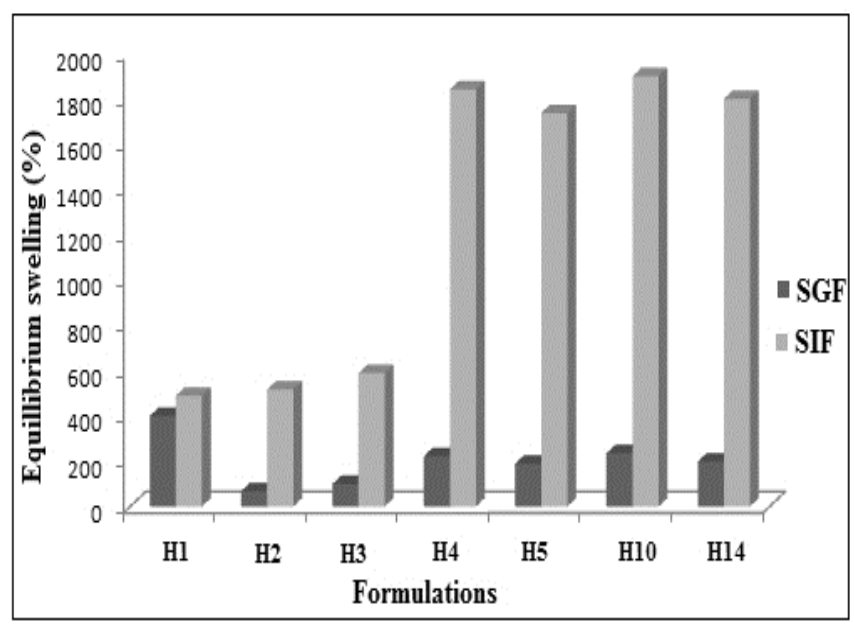

Figure 6. Equilibrium swelling (\%) of selected formulations in biological media. SGF: Simulated Gastric fluid; SIF: Simulated Intestinal Fluid

\subsection{Drug entrapment efficiency (\%)}

Entrapment efficiency is the amount of added drug (in percent) that is encapsulated in the formulation (Table II). Among the selected formulations, formulation $\mathrm{H} 10$ found to have higher VAL loading and entrapment efficiency. This might be due to comparatively higher swelling capacity of the formulation H10. Low coefficient of variance $(<2.5 \%)$ in $\%$ VAL entrapment indicates uniformity of drug entrapment in different batches.

Table II VAL loading into CGN-g-Poly (AM-co-IA) formulations, Hydrolyzed collagen: $\left(\begin{array}{ll}1.0 & \mathrm{~g}\end{array}\right)$; acrylamide: $1.0 \mathrm{~g}, \mathrm{H} 2 \mathrm{O}: 30 \mathrm{ml}$, temperature: $80{ }^{\circ} \mathrm{C}$

\begin{tabular}{|c|c|c|}
\hline Polymer Code & $\begin{array}{c}\text { \%VAL } \\
\text { loading }\end{array}$ & $\begin{array}{c}\text { \%VAL } \\
\text { entrapment }\end{array}$ \\
\hline H4 & $96 \pm 2.0$ & $79 \pm 2.1$ \\
\hline H10 & $\mathbf{9 8} \pm \mathbf{2 . 3}$ & $\mathbf{8 3} \pm \mathbf{2 . 5}$ \\
\hline H11 & $95 \pm 1.9$ & $76 \pm 1.7$ \\
\hline
\end{tabular}




\subsection{In vitro drug release study}

In vitro VAL release from CGN-g-Poly (AMco-IA) hydrogel systems were evaluated in $\mathrm{pH}$ progressive media i.e. SGF as well as in SIF as the dissolution medium to see the release behavior in different $\mathrm{pH}$ conditions. The applied formulative variable i.e. change in monomer ratio, concentration of the crosslinker in the crosslinked polymer was compared for their influence on drug release rate. Formulation H11 showed slowest drug release profile in both the medium than other formulations and formulation $\mathrm{H} 10$ showed relatively more release in both the media (Fig. 7). The crosslinking between the polymer networks found to play major role in the release of VAL. The release of VAL from the hydrogel system was also found to be dependent on swelling properties of the hydrogel in particular medium. Comparatively much higher VAL release was observed in SIF than that in SGF. This may be due to the higher swelling capacity of the system in SIF. VAL release from hydrogel was found to be controlled up to $24 \mathrm{~h}$.

\subsection{In vitro drug release kinetic mechanism}

To determine the mechanism of drug release from CGN-g-P (AM-co-IA) matrices, different kinetic models like zero order kinetic, first order kinetic, Higuchi model were used. Regression coefficient $\left(\mathrm{R}^{2}\right)$ values of each kinetic model were compared to find out the best fit model. By comparing the $\mathrm{R}^{2}$ values of different models, Higuchi model was found to be best fit (Table III). So it could be predicted that release of VAL from the hydrogel formulations were of diffusion type. After storing the formulations for three months at accelerated stability condition i.e. $40^{\circ} \mathrm{C} / 75 \% \mathrm{RH}$ as per I.C.H. guidelines, the VAL loaded hydrogel were found to retain the same drug content with minor deviations

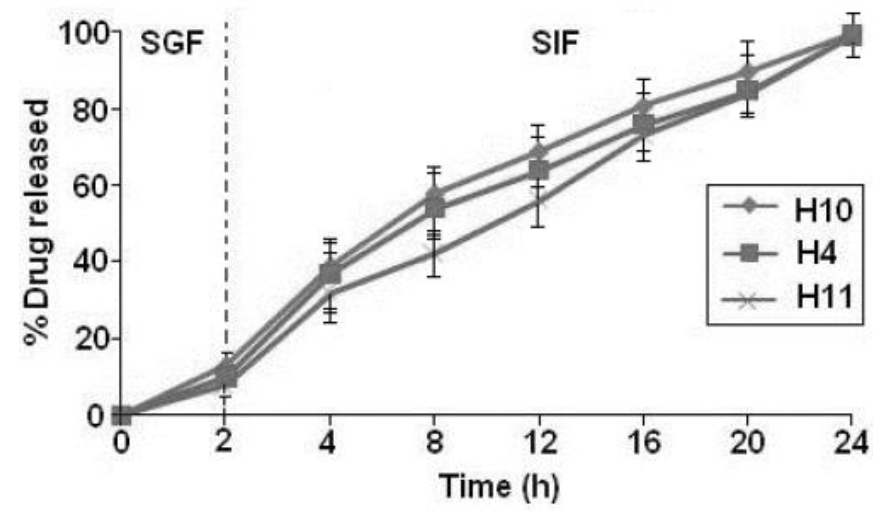

Figure 7 Comparison of \% VAL release from VAL loaded formulations ( $\mathrm{H} 4, \mathrm{H} 10$ and $\mathrm{H} 11)$ in $\mathrm{pH}$ progressive biological media

Table III. Fitting results of experimental VAL release data of CGN-g-P (AM-co-IA) hydrogel formulations H4, H10 and H11 to different kinetic equations.

\begin{tabular}{|c|c|c|c|c|c|c|}
\hline Formulation & $\begin{array}{c}\text { Zero order } \\
\left(\mathbf{k}_{\mathbf{0}}\right)\end{array}$ & $\mathbf{R}^{\mathbf{2}}$ & $\begin{array}{c}\text { First order } \\
\left(\mathbf{k}_{\mathbf{1}}\right)\end{array}$ & $\mathbf{R}^{\mathbf{2}}$ & $\begin{array}{c}\text { Higuchi } \\
\left(\mathbf{k}_{\mathbf{H}}\right)\end{array}$ & $\mathbf{R}^{\mathbf{2}}$ \\
\hline $\mathrm{H} 4$ & 9.521 & 0.9302 & 0.714 & 0.9854 & 59.475 & $\mathbf{0 . 9 8 6 8}$ \\
& $(1.650)$ & $(0.090)$ & $(0.109)$ & $(0.015)$ & $(1.371)$ & $\mathbf{( 0 . 0 0 1 )}$ \\
\hline \multirow{2}{*}{$\mathrm{H} 10$} & 7.965 & 0.9604 & 0.049 & 0.9410 & 21.341 & $\mathbf{0 . 9 8 8 4}$ \\
& $(1.446)$ & $(0.061)$ & $(0.124)$ & $(0.011)$ & $(1.423)$ & $\mathbf{( 0 . 0 0 6 )}$ \\
\hline \multirow{2}{*}{$\mathrm{H} 11$} & 8.634 & 0.9831 & 0.078 & 0.9280 & 24.142 & $\mathbf{0 . 9 7 9 2}$ \\
& $(1.244)$ & $(0.018)$ & $(0.191)$ & $(0.024)$ & $(1.482)$ & $\mathbf{( 0 . 0 0 9}$ \\
\hline
\end{tabular}




\section{Conclusion}

In this study, a series of hydrogels based on CGN, AM and IA was prepared through free radical polymerization. These $\mathrm{pH}$ sensitive gels respond to small change of $\mathrm{pH}$ to much sharper extent than other $\mathrm{pH}$-sensitive gels, which may be due to the presence of two carboxylic groups of IA in the hydrogels. Hydrogels with high content of itaconic acid showed more drug release than those gels with low content of IA. A negligible amount of VAL is released in SGF while in SIF more than $80 \%$ of the total drug is released. From the in vitro drug release study in $\mathrm{pH}$ progressive media, formulation $\mathrm{H}_{10}$ showed comparatively higher release extending up to $24 \mathrm{~h}$. From the drug release kinetic study, Higuchi model was found to be best fit among all the models. The mechanism of VAL release from the hydrogel matrix was found to be of diffusion type. To be used for biomedical applications, the amount of the residual monomers in the prepared hydrogel must be kept as low as possible. So we used LCMS/MS and HPLC technique respectively to detect and quantify the residual $\mathrm{AM}$ and IA in the synthesized hydrogel. In addition, it is expected that the resulted porous polymers show more compatibility with body when they use as drug delivery systems, because of use of protein as a natural backbone. These results indicate that CGN-g-P- (AM-co-IA) can be used as the sustained-release carrier of VAL in oral administration.

\section{References}

[1] Bajpai, A.K.; Sandeep K.S.; Bhanu, S.; Kankane, Responsive polymers in controlled drug delivery, S. Prog Polym Sci 2008, 33, 1088. DOI: 10.1016/j.progpolymsci.2008.07.005

[2] Deligkaris, K.; Tadele, T. S.; Olthuis, W.; Van den Berg, A. Hydrogel-based devices for biomedical applications, Sens Act B chem 2010, $147, \quad 765 . \quad$ DOI: 10.1016/j.snb.2010.03.0853

[3] Huynh, D. P.; Huynh, C. T.; Lee, D. S. Picolyamine based $\mathrm{pH} /$ temperature sensitive hydrogels, Macromol Res 2010, 18, 589. DOI: 10.1007/s13233-010-0614-X

[4] Wang, Q.; Xie, X.; Zhang, X.; Zhang, J.; Wang, A. Preparation and swelling properties of $\mathrm{pH}$-sensitive composite hydrogel beads based on chitosan- $g$-poly (acrylic acid)/vermiculite and sodium alginate for diclofenac controlled release, Int J Biol Macromol 2010, 46, 356. DOI: 10.1016/j.ijbiomac.2010.01.00x9

[5] Hong, K. H.; Jeon, Y.S.; Chung D. J.; Kim, J.H. Drug release characteristics of modified PHEMA hydrogel containing thermoresponsive pluronic copolymer,Macromol Res 2010, 18, 204. DOI: 10.1007/s13233009-0129-5

[6] Gils, P.S.; Ray, D.; Sahoo, P.K.; Controlled Release of Doxofylline from Biopolymer Based Hydrogels, Am. J. Biomed. Sci. 2010, 2(4), 373-383. DOI: 10.5099/aj100400373

[7] LjTomic', S.; Mic' ic', M. M.; Dobic', S. N.; Filipovic, ' J. M.; Suljovrujic,' E. H. Smart poly (2-hydroxyethyl methacrylate/ itaconic acid) hydrogels for biomedical application, Rad Phys Chem 2010, 79, 643. DOI: 10.1016/j.radphyschem.2009.11.015

[8] Milašinović, N.; Milosavljević, N.; Filipović, J.; Knežević-Jugović Z.; Kalagasidis Krušić, Synthesis, characterization and application of poly( $N$-isopropylacrylamide-co-itaconic acid) hydrogels as supports for lipase immobilization, M. React Funct Polym 2010, 70, 807. DOI: 10.1016/j.reactfunctpolym. $\underline{2010.07 .017}$

[9] Pourjavadi, A.; Salimi, H. New Protein-Based Hydrogel with Superabsorbing Properties: Effect of Monomer Ratio on Swelling Behavior and Kinetic, Ind Eng Chem Res 2008, 47, 9206. DOI: 10.1021/ie800247

[10] Betancourt, T.; Pardo, J.; Soo, K.; Peppas, N. A. Characterization of $\mathrm{pH}$-responsive hydrogels of poly(itaconic acid- $g$-ethylene glycol) prepared by UV-initiated free radical polymerization as biomaterials for oral delivery of bioactive agents, $\mathrm{J}$ Biomed Mat Res Part A 2010, 93A, 175. DOI: $10.1002 / \mathrm{jbm} . \mathrm{a} .32510$

[11] Nayak, U.Y.; Shavi, G. V.; Nayak, Y.; Averinen, R. K.; Mutalik, S.; Reddy, S. M.; 
Gupta P. D.; Udupa, N. Chronotherapeutic drug delivery for early morning surge in blood pressure: A programmable delivery system, J. Control Rel 2009, 136, 125. DOI: 10.1016/j.jconrel.2009.02.008

[12] MacDonald, Russell J, Polymers prepared from concentrated solutions of $\mathrm{N}^{\prime}, \mathrm{N}^{\prime}$ methylene- bisacrylamide, USPatent 5354903 (1994)

[13] Xiaohua, Q.; Mingzhu L.; Zhenbin, C. Fen, Z. Study on the swelling kinetics of superabsorbent using open circuit potential measurement, Euro Polym J 2008, 44, 743. DOI: 10.1016/j.eurpolymj.2007.12.010

[14] Carstensen, J.T.; Rhodes, C.T. Drug stability, Principles and Practices; Marcel Dekker: New York, 2000.
[15] Isıklan, N.; Kursun, F.; Inal, M. Graft copolymerization of itaconic acid onto sodium alginate using benzoyl peroxide, Carbohydr Polym 2010, 79, 665. DOI: 10.1016/j.carbpol.2009.09.021

[16] Li, W.W.; Li, H.; Liu Z.F.; Qiao, Q. Determination of residual acrylamide in medical polyacrylamide hydrogel by high performance liquid chromatography tandem mass spectroscopy, Biomed Environ Sci 2009, 22, 28. 